\title{
Superradiant Cancer Hyperthermia using a Buckyball Assembly of Quantum Dot Emitters
}

\author{
Sudaraka Mallawaarachchi, Student Member, IEEE, Malin Premaratne, Fellow, OSA, and Philip K. Maini
}

\begin{abstract}
With the emergence of nanomedicine, targeted hyperthermia has a high potential of becoming a first-line cancer treatment modality. However, hyperthermia needs to be precisely controlled to avoid damaging adjacent healthy tissues. Due to the uncontrollable transfer of heat from the tumor boundary to healthy tissues, it is extremely difficult to control the temperature increase. As a solution, in this paper, we propose using a superradiant emitter assembly to deliver an ephemeral and powerful thermal pulse to enhance cancer hyperthermia by reducing damage to healthy tissues. Our assembly comprises quantum dot emitters arranged in the shape of a buckyball. We obtain criteria for our assembly to be superradiant and prove that it is possible to control superradiance using an external electric field. We analytically obtain expressions for the assembly dynamics and conduct thermal studies using a simple breast cancer model constructed using experimental parameters. Our results indicate that using a series of superradiant pulses can enhance cancer hyperthermia by minimizing the damage to adjacent healthy tissues.
\end{abstract}

Index Terms-Coupled mode analysis, nanotechnology, photon radiation effects

\section{INTRODUCTION}

$\mathbf{H}$ YPERTHERMIA refers to the cytotoxic increase of body temperature beyond its normal value. The effects of hyperthermic cytotoxicity are known to intensify in deoxygenated and acidic cellular environments, establishing its potency as an ideal cancer treatment modality. The clinical appeal of hyperthermia is further enhanced by the fact that it does not involve using harmful chemicals or radiation. However, high temperature exposure causes protein denaturation in healthy and malignant cells alike. Therefore, caution must be exercised in clinical hyperthermia to minimize potential harm to healthy cells [1]. Fortunately, the advent of nanomedicine has paved the way to targeted treatment modalities that facilitate the direct delivery of thermal energy to malignant cells. This spares healthy tissues from significant heat exposure [2]. However, even targeted heating of cancer cells will generate undesired results due to heat propagation, which leads to induced hyperthermia of neighboring healthy cells. The emergence of nanobiophotonics, which is the amalgamation between biomedical engineering and nanophotonics, presents us with a myriad of control techniques for heat propagation

S. Mallawaarachchi and M. Premaratne are with the Advanced Computing and Simulation Laboratory $(\mathrm{A} \chi \mathrm{L})$, Department of Electrical and Computer Systems Engineering, Monash University, Victoria 3000, Australia (e-mail: sudaraka@ieee.org; malin.premaratne@monash.edu).

P.K. Maini is with the Wolfson Centre for Mathematical Biology, Mathematical Institute, Oxford University, Woodstock Road, Oxford OX2 6GG, United Kingdom (e-mail: maini @maths.ox.ac.uk)

Manuscript received May 25, 2018 and delivery. The utilization of such methods could provide us with the means to tackle these complicated issues and aid in clinically establishing cancer hyperthermia as a first-line treatment modality.

In nanophotonics, spontaneous emission of photons from excited emitter assemblies is generally regarded without involving the contributions from inter-emitter interactions. In such scenarios, the emission linearly scales with the number of system emitters, $N$ [3]. However, under certain stringent conditions, the involvement of inter-emitter interactions gives rise to various interesting quantum electrodynamic (QED) phenomena such as Fano interferences, spasing, chemical interface damping and superradiance [4]-[8]. In particular, the effect of superradiance has the potential to scale the emission quadratically with the number of system emitters $\left(N^{2}\right)$ and curtail the emission time by a factor of $(1 / N)$, metamorphosing the emission to an ephemeral pulse comprising a large amount of energy. If controlled, the nature of these pulses should cause hyperthermia in adjacent malignant cells while sparing the heat propagation into neighboring healthy cells.

Numerous efforts have been made at observing, understanding and controlling superradiance [9], [10]. Unfortunately, superradiance in its raw form is a single-occurring event, and therefore is of minute practical significance. However, successful attempts have been made recently at sustaining superradiance in the steady-state [11], which has enabled its utilization in fields such as energy harvesting, biomedical engineering and lasing [12]-[14]. Therefore, a superradiant emitter assembly for cancer hyperthermia is an intriguing concept that is worthwhile exploring.

Although a conceptual proposal of utilizing superradiance for cancer hyperthermia is unequivocally sound, validation of such an assembly is highly challenging. For instance, although the characteristics of superradiance and its sustenance have been studied in great detail, few attempts have been made at validating the structural requirements of an emitter assembly to be superradiant. It is well known that the existence of superradiance is strictly contingent on fulfilling two superradiant criteria (SRC); namely size and symmetry [3]. These SRC require strict geometric distributions for the emitters and significantly limit the number of emitters that can partake in superradiant emission. In order to propose using superradiance for cancer hyperthermia, it is necessary to validate that the assembly fulfills SRC. Furthermore, it should be possible to involve a large number of emitters in order to fulfill the power requirements for cancer hyperthermia.

In this paper, we propose delivering cancer hyperthermia using an emitter assembly comprising quantum dots (QDs), 


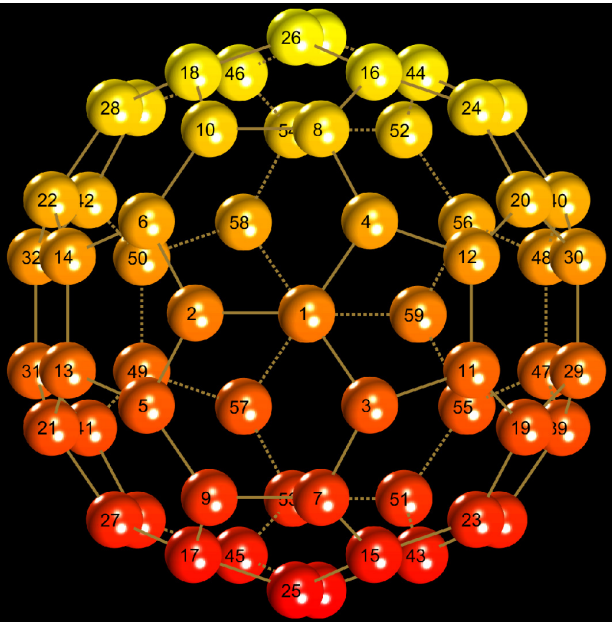

Fig. 1. Proposed superradiant emitter assembly configuration where 60 QDs are placed at the vertices of a truncated icosahedron. The assembly is designed nanoscopically to fulfill the size SRC, where the emitter dimensions $(r)$ are much smaller than the wavelength with which it interacts $(\lambda)$. Furthermore, each QD is assumed to comprise a permanent dipole and an external control field is used to manipulate the behavior of the resultant dipole of each QD. Under the correct conditions, this assembly fulfills the symmetry SRC as well.

placed at the vertices of a buckyball, as shown in Fig. 1. This shape, formally known as a truncated icosahedron, is the well-known geometry of the $\mathrm{C}_{60}$ buckminsterfullerene [15]. The radiative coupling nature of QD ensembles is experimentally proven and the observed broad spectrum emission characteristics make them ideal for cancer hyperthermia [16]. Furthermore, QD assemblies have previously been used for in-vitro hyperthermia with success [17]. Moreover, the fact that numerous QD types have proven biocompatible characteristics undoubtedly proves their suitability for this application [18]. Furthermore, recent advances in designing complex QD structures strongly bolsters the possibility of realizing our proposed shape in an experimental setting [19], [20]. We propose using an external control field to alter the resultant dipole characteristics of the QDs as a mechanism to control superradiant emission. Furthermore, we perform a complete spectral analysis of the assembly to assess its emission dynamics. Then we use realistic parameters for breast cancer and healthy tissues to conduct thermal simulations [21]. Our results prove that the proposed emitter assembly is capable of delivering superior cancer hyperthermia compared to conventional models that do not incorporate inter emitter interactions.

\section{Methods}

\section{A. Quantum Dot Emitter Assemblies}

We begin our analysis by considering the emission of a general assembly of $N$ QDs without adhering to any geometric constraints. We approximate each QD by a two-level dipole and assume that at $t=0$, the entire assembly is at the fully excited stage. Now we model the decaying behavior of the assembly in two configurations.
1) Non-interacting assemblies: Assuming that the QDs are placed sufficiently far away from each other, there will be no inter-emitter interactions. This is the conventional arrangement and corresponds to the typical scenario of spontaneous emission. The emission of each photon is governed by the single QD spontaneous emission rate, denoted by [3]:

$$
\Lambda_{a t}=\frac{8 \pi^{2}|\mathbf{q}|^{2}}{3 \epsilon_{b} \hbar \lambda^{3}},
$$

where $|\mathbf{q}|$ is the norm of the dipole vector (dipole matrix element), $\epsilon_{b}$ is the bath permittivity, $\hbar$ is the reduced Planck constant and $\lambda$ is the emitted wavelength.

Let $\mathcal{P}_{\omega}$ denote the power emitted by the entire assembly at frequency $\omega$. Now, without loss of generality, it is possible to approximate the emission characteristics for the assembly using [22]:

$$
\tilde{\mathcal{P}}_{\omega, t} \approx N \exp \left(-t / \Lambda_{a t}\right) \mathcal{P}_{\omega}
$$

Equation (2) describes an exponentially decaying pulse of energy, the frequency characteristics of which are completely governed by the power spectrum of the emitter assembly.

2) Superradiant assemblies: An emitter assembly needs to adhere with two SRC in order to exhibit superradiant characteristics. These SRC are discussed in detail in section II-B. Without loss of generality, we now assume that our QD assembly fulfills these criteria. Due to superradiance, the emitted pulse will scale quadratically and its duration will scale inversely with the number of emitters [22]:

$$
\tilde{\mathcal{P}}_{\omega, t} \approx N^{2} \exp \left(-t /\left(N \Lambda_{a t}\right)\right) \mathcal{P}_{\omega}
$$

Once the spectral characterization is complete, equations (2) and (3) will allow us to simulate and compare the emission dynamics of these two emitter assemblies.

\section{B. Superradiant Criteria}

Superradiance is a well-studied phenomenon that has attracted attention due to its unique temporal and spectral characteristics. There are strict requirements to which an emitter assembly must adhere, in order to depict superradiance.

1) Size SRC: This SRC is intuitive; when the linear size of the assembly $(\mathcal{S})$ is much smaller than the wavelength with which it interacts $(\mathcal{S} \ll \lambda)$, the emitters become indiscernible to the emitted energy. Therefore, rather than functioning as individual emitters, the entire assembly functions as a global radiating dipole. This phenomenon is fundamental to superradiance. Therefore, any emitter assembly confined within subwavelength volumes fulfills the size criterion and is a potential candidate for superradiance.

2) Symmetry SRC: This SRC depends on the form of interemitter interactions. For QDs that are sufficiently proximal to each other, but not close enough for Förster type coupling or tunneling, it is possible to assume that Van der Waal's coupling is the main form of inter-emitter photon transfer. In order to understand the behavior of this coupling, it is necessary to begin with fundamental QED theory to compose and solve the master equation for the superradiant system. This lengthy derivation is detailed elsewhere in the literature [3]. 


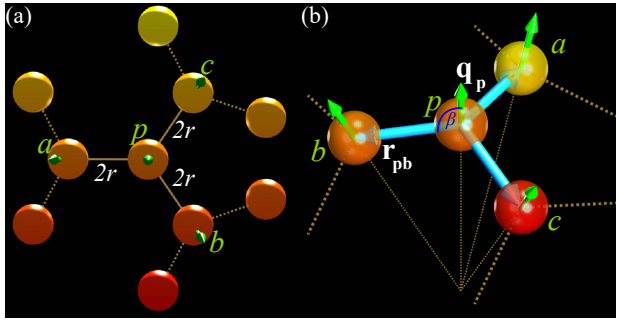

Fig. 2. (a) Each QD in the assembly (say $p$ ) is assumed to interact with 3 nearest neighbors $(a, b, c)$, each at a distance of $2 r$. Interactions beyond this limit can be safely ignored for sufficiently large $r$. (b) The symmetry SRC of the assembly depends on the dot product quantity: $\hat{\mathbf{q}}_{p} \cdot \mathbf{r}_{p b}$, the value of which relies on the shown inter-vector angle $\beta$. Due to the symmetry of a truncated icosahedron, this angle is constant for all QDs across the assembly. This implies that the model is capable of fulfilling the symmetry SRC.

The imaginary part of the master equation presents the following expression for inter-emitter Van der Waal's interactions between the QDs $p$ and $b$ as shown in Fig. 2a:

$$
\Lambda_{i n t}(p, b)=\frac{\left|\mathbf{q}_{p}\right|^{2}}{4 \pi \epsilon_{b} \hbar} \frac{1}{r_{p b}^{3}}\left[1-3 \frac{\left(\hat{\mathbf{q}}_{p} \cdot \mathbf{r}_{p b}\right)^{2}}{\left|\mathbf{r}_{p b}\right|^{2}}\right]
$$

where $\hat{\mathbf{q}}_{p}$ denotes the resultant unit dipole vector of QD $p$. The terms $\mathbf{r}_{p b}$ and $\left|\mathbf{r}_{p b}\right|$ denote the interaction vector between the QDs and its norm, respectively. This quantity is commonly referred to as the hopping-interaction strength because it defines the virtual photon exchange rate among emitters [9].

The dot product term: $\hat{\mathbf{q}}_{p} \cdot \mathbf{r}_{p b}$, as shown in Fig. $2 \mathrm{~b}$, is pivotal to the symmetry SRC. An assembly of QDs must maintain this coupling rate at a uniform value across all interacting QDs. If this criterion does not hold, then the emitters will couple with each other at different rates and superradiance will cease to exist. An arbitrary geometric distribution of QDs will obviously breach the symmetry $\mathrm{SRC}$ because $\Lambda_{\text {int }}(p, b)$ will take arbitrary values for different interacting QDs, depending on the inter-emitter distances and their unique resultant dipoles. This clearly implies that the symmetry SRC cannot be guaranteed for an arbitrary geometric distribution of interacting QDs. However, a symmetric ring or an equidistant linear QD configuration clearly satisfies the SRC, since it is possible to maintain $\hat{\mathbf{q}}_{p} \perp \mathbf{r}_{p q} \Longrightarrow \hat{\mathbf{q}}_{p} \cdot \mathbf{r}_{p q}=0, \forall p, q$, which explains the popularity of these geometries in the literature [23], [24]. However, it is well-known that such structures support superradiant emission only up to a few emitters. This is clearly a limitation and will hinder the practical applicability of superradiance [9].

Furthermore, it is hypothesized that an assembly maintaining superradiant criteria should also exhibit superabsorption characteristics due to quantum time reversal symmetry [9]. This implies that the proposed assembly should theoretically depict an analogous enhancement in the initial heating process involving the absorption of electromagnetic radiation.

Next we show that our proposed emitter assembly can fulfill both these SRC while maintaining superradiance for an assembly of 60 QDs.

\section{Proposed Emitter Assembly}

The emitter assembly proposed in Fig. 1 comprises 60 QDs that are placed at the vertices of a truncated icosahedron. The QDs are positioned according to Cartesian coordinates that are computed using the well-known even permutations method. Furthermore, the QDs are labeled using the numbering scheme based on permutations in the alternating group $A_{5}$ [15].

1) Maintaining size $S R C$ : When constructed using a scaling dimension (say $r$ ), all vertices of the truncated icosahedron will lie on a sphere with radius: $r \sqrt{9 \psi+10}$, where $\psi$ is the golden mean and the edge length will be $2 r$ [15]. This implies that $r_{p b}=2 r$, where QD $b$ is a nearest neighbor of $p$. As shown in Fig. 2a, we assume that each QD will have exactly 3 nearest neighbors, each at a distance of $2 r$. This is a fair assumption, given that the hopping-interaction strength is proportional to $1 / r_{p q}^{3}$ and within nanoscopic dimensions, interactions beyond the nearest neighbor limit are usually safely ignored [3], [9], [10]. It is noted that by choosing $r$ appropriately, our model can be designed to fulfill the size SRC. Furthermore, by varying the QD dimensions while maintaining the size SRC, it will be possible to tune the emission characteristics due to the resulting changes to QD parameters such as absorption and emission.

2) Maintaining symmetry SRC: Assuming that it is possible to obtain a geometrical QD assembly where the resultant polarization vector of each QD intersects with the origin of the truncated icosahedron and the center of the respective $\mathrm{QD}$, it is possible to maintain the SRC. In this configuration, each QD will have a unique unit polarization vector. As shown in Fig. 2b, under this assumption, the angle $\beta$ then determines the value of the hopping-interaction strength in equation (4). Simple trigonometry shows that $\Lambda_{\text {int }}(p, b)=0.8778616\left|\mathbf{q}_{p}\right|^{2} / 4 \pi \epsilon_{b} \hbar r^{3}$ is a constant where $b$ is a nearest neighbor of $p$. Maintaining a constant value for $\Lambda_{\text {int }}(p, b), \forall(p, b)$, where $b$ is a nearest neighbor of $p$, is clearly in agreement with the symmetry SRC.

Therefore, our proposed emitter assembly should be superradiant under these conditions. However, it is noted that by using non-spherical QDs, the symmetry SRC is potentially challenged due to anisotropy and the existence of multiple resonant modes.

\section{Superradiant Controllability}

Now we propose a method to realistically achieve the resultant dipole arrangement to maintain the symmetry SRC. It is known that QDs built using specific material and size criteria possess intrinsic, permanent dipoles of high potentials [25][27]. Furthermore, it is well-known that the application of an external electric field will induce a dipole moment due to the polarizability of the exciton. Fig. 3a depicts this scenario. Here we have shown the field-induced dipoles using red arrows. Given the nanoscopic dimensions of the emitter assembly, we have assumed that all QDs uniformly interact with the incident electric field. The white arrows denote the direction of the permanent dipoles of each QD. When two dipole moments are present within the same QD, the resultant dipole moment is the pivotal parameter controlling inter-emitter couplings. The 
resultant dipole moment for each QD is shown by the green arrows in Fig. 3b.

Under this configuration, superradiant emission of the proposed assembly can clearly be controlled by alternating the control field. When the control field is OFF, the resultant dipole will be the permanent dipole for each QD and the symmetry SRC is clearly violated. Emission will still exist but without superradiant enhancement. When the control field is $\mathrm{ON}$, the induced dipoles will change the resultant dipole to the configuration in Fig. 3b. This will reinstate the superradiant behavior to the assembly. When superradiant, the system will emit a high burst of energy within a very short time. Alternating the control field will result in pulsated emission of high energy, ephemeral pulses. It is noted that this controllable pulsating behavior is not present in conventional, non-interacting emitter assemblies.

However, in a purely practical perspective, arranging a QD assembly to perfectly meet the presented mathematical requirements will be challenging. However, recent advancements in QD technology strongly suggests the possibility of realizing the structure [19], [20]. Furthermore, it is noted that the value of $\Lambda_{\text {int }}(p, b)$ beyond the nearest neighbor condition is not a constant under our configuration. However, in a practical setting, the presence of such trivial interactions will not hinder the effects of superradiance [28].

Assuming all these criteria are maintained, the next step is to study the spectral characteristics of the QD emitter assembly. Once the spectra are known, it is possible to use equations (2) and (3) to conduct simulations and study the dynamics of the proposed assembly.

\section{E. Spectral Characterization}

Any emitter assembly should be benchmarked using spectral analysis in order to ascertain its power delivering capabilities. Without loss of generality, coupled mode theory presents a method to directly express the fluctuations of electric energy within the emitters of the assembly [29].

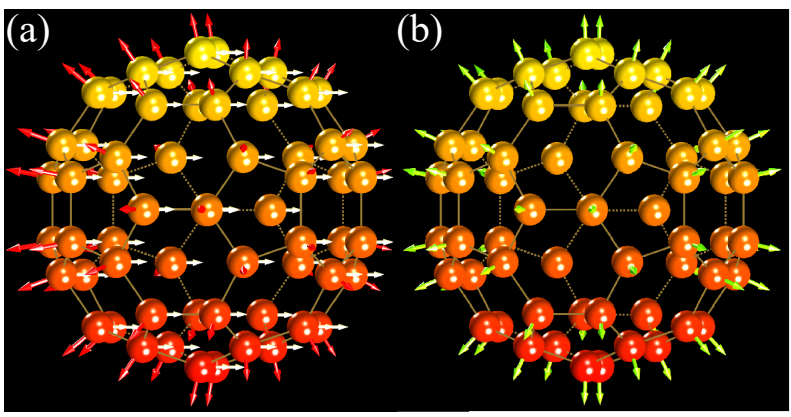

Fig. 3. (a) An assembly of QDs with large, intrinsic, permanent dipoles (white arrows) placed at the vertices of a truncated icosahedron. When the assembly is exposed to an electric field, a temporary dipole is induced (red arrows) within the QDs. Since the assembly is minuscule, it is assumed that each QD interacts with the incident field in the same manner. Therefore, the induced dipoles have the same magnitude and direction. (b) The resultant dipole moments for the two dipoles are shown by arrows. When the permanent and induced dipoles are perfectly matched, it is possible to have all resultant dipoles originating from the center of the buckyball and traverse through the center of the corresponding QD.

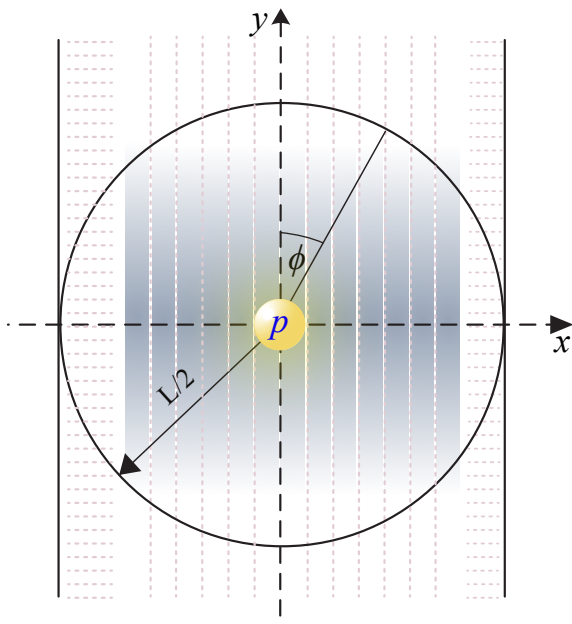

Fig. 4. This diagram illustrates the coupling of emission from the $p$ th QD with free space. For analysis, the free space is first confined to a width of $L$ and the vertical region is divided into $2 m+1$ radiation channels where $m=L / \lambda_{0}$. A circular geometry of radius $L / 2$ is selected around the $p$ th emitter. Each channel can now be uniquely identified using the vertical angle $\phi$. Realistic free space conditions are later achieved by applying the limit $L \rightarrow \infty$.

1) Coupled mode treatment: Let the normalized, time and frequency varying, electric field amplitudes within the QD emitters be denoted by: $\mathbf{a}(\omega, t)=\left[a_{1}(\omega, t) \ldots a_{N}(\omega, t)\right]^{T}$. Then the system dynamics are captured using the simple expression:

$$
\frac{\partial}{\partial t} \mathbf{a}(\omega, t)=\left[j \boldsymbol{\Omega}_{0}-\boldsymbol{\Lambda}\right] \mathbf{a}(\omega, t)+\diamond(\omega, t),
$$

where $\boldsymbol{\Omega}_{0}=\operatorname{diag}\left[\omega_{0}^{1} \ldots \omega_{0}^{N}\right]$ contains the resonant frequencies of each emitter. The $N \times N$ matrix $\boldsymbol{\Lambda}=\boldsymbol{\Lambda}_{A}+\boldsymbol{\Lambda}_{E}+\boldsymbol{\Lambda}_{\text {int }}$, encodes the rates of photon absorption: $\boldsymbol{\Lambda}_{A}=\operatorname{diag}\left[\Lambda_{A}^{1} \ldots \Lambda_{A}^{N}\right]$ and emission: $\boldsymbol{\Lambda}_{E}=\operatorname{diag}\left[\Lambda_{E}^{1} \ldots \Lambda_{E}^{N}\right]$ of all emitters. The interemitter coupling rates are encoded within $\boldsymbol{\Lambda}_{\text {int }}$ and takes the form of equation (4). Generally, the $N \times 1$ matrix $\diamond(\omega, t)$ expresses the photon generation rate within emitters [29].

For the case of QDs operating in the thermal regime, we assume that each emitter is modeled based on a graybody radiator. Since each source emits in accordance with the fluctuation dissipation theorem, and assuming a stationary characteristic for each source, $\diamond(\omega, t)$ converts to the time invariant form: $\diamond(\omega)=\sqrt{\boldsymbol{\Lambda}_{A}} \mathbf{n}(\omega)$. Now, the $N \times 1$ matrix $\mathbf{n}(\omega)$ has the form [30]:

$$
\left\langle n_{p}^{*}(\omega) n_{b}(\omega)\right\rangle=\Theta(\omega, T) \delta\left(\omega-\omega^{\prime}\right) \delta_{p b},
$$

where $\Theta(\omega, T)=\varepsilon_{\omega} \hbar \omega /\left(2 \pi \exp \left(\hbar \omega / k_{B} T\right)\right)$ is the wellknown Planck energy of thermal photons radiating at temperature $T$ and angular frequency $\omega$. Here, $\varepsilon_{\omega}$ and $k_{B}$ denote the emissivity of the QDs and the Boltzman constant, respectively.

Equation (5) describes the temporal and spectral dynamics of our system, the characteristics of which are fully defined using photon couping rates. In order to conduct simulations, it is necessary to establish analytical relationships between them.

It is obvious that all generated photons of the assembly must either be absorbed within the generated emitter, coupled to free space or coupled to other emitters. Furthermore, due to energy conservation, all emitted photons from the system must 
either be coupled to other emitters or free space. Considering only the emitted photons, equation (5) modifies to $\frac{\partial}{\partial t} \mathbf{a}(\omega, t)=$ $\left[j \boldsymbol{\Omega}_{0}-\left(\boldsymbol{\Lambda}_{E}+\boldsymbol{\Lambda}_{\text {int }}\right)\right] \mathbf{a}(\omega, t)$, which yields:

$$
\frac{\partial}{\partial t} \mathbf{a}^{*} \mathbf{a}=\mathbf{a}^{*}\left[-2\left(\boldsymbol{\Lambda}_{E}+\boldsymbol{\Lambda}_{i n t}\right)\right] \mathbf{a} .
$$

The negative sign implies that the process is an emission.

We continue our analysis by focusing on a finite region of free space of length $L$ around an arbitrary emitter $p$, as shown in Fig. 4. This region is quantified into $2 m+1$ vertical radiation channels, where $m=L / \lambda_{0}$. Since all these photons are emanating from the set of emitters, energy conservation yields:

$$
2\left(\boldsymbol{\Lambda}_{E}+\boldsymbol{\Lambda}_{i n t}\right)=\left[\boldsymbol{\Lambda}_{E}^{\forall p \rightarrow \forall \phi}\right]^{T}\left[\boldsymbol{\Lambda}_{E}^{\forall p \rightarrow \forall \phi}\right],
$$

where $\boldsymbol{\Lambda}_{E}^{\forall p \rightarrow \forall \phi}$ is the rate matrix for all emitters coupling to all radiation channels.

To obtain a relationship for $\boldsymbol{\Lambda}_{E}^{\forall p \rightarrow \forall \phi}$, we analyze coupling between the arbitrary $p$ th emitter shown in Fig. 4 and free space. We assume that the $p$ th QD is an isotropic radiator and the emission is distributed across the vertical radiation channels. For mathematical convenience, we begin by analyzing the radiation channels confined within a circular geometry of radius $L / 2$. Now, each channel can be uniquely characterized by the vertical angle $(\phi)$.

The photon emission rate from the $p$ th QD can be expressed as: $\Lambda_{E}^{p}=2 \sum_{\phi=-\pi / 2}^{p i / 2} \Lambda_{E}^{p \rightarrow \phi}$. Here, the term $\Lambda_{E}^{p \rightarrow \phi}$ denotes the photon emission rate to channel $\phi$. The rate to each channel can be projected onto the perpendicular direction by using the relationship: $\Lambda_{E}^{p \rightarrow \perp}=\Lambda_{E}^{p \rightarrow \phi} \cos \phi$. Hence,

$$
\Lambda_{E}^{p}=2 \Lambda_{E}^{p \rightarrow \perp} \sum_{-\frac{\pi}{2}<\phi<\frac{\pi}{2}} \frac{1}{\cos \phi} .
$$

Note that $\phi$ can only assume discrete and realistic values corresponding to channels in Fig. 4. Applying trigonometric relations and the limit $L \rightarrow \infty$ to equation (9) yields: $\Lambda_{E}^{p}=2 \Lambda_{E}^{p \rightarrow \perp} m \pi$. Based on this result, it is possible to obtain the following relationship for the emitter-channel coupling: $\Lambda_{E}^{p \rightarrow \phi}=\Lambda_{E}^{p} \sec \phi / 2 m \pi$. This is the general form of an element in matrix $\Lambda_{E}^{\forall p \rightarrow \forall \phi}$. Substituting this result in equation (8) directly yields:

$$
\left[\boldsymbol{\Lambda}_{i n t}\right](p, q)=\frac{1}{2} \sqrt{\Lambda_{E}^{p} \Lambda_{E}^{q}}\left(1-\delta_{p, q}\right),
$$

which is the sought after relationship to completely define the emitter assembly with measurable photon coupling rates. It is noted that since only the nearest neighbor interactions are used in our model, $\boldsymbol{\Lambda}_{i n t}$ is a sparse matrix as shown in Fig. 5.

2) Analytical emission spectrum: Fourier transformations and the framework of the fluctuation dissipation theorem for thermal emission can be used to establish an analytical expression for the spectrum. We have published a lengthy and descriptive derivation of the analytical emission spectrum previously [31], [32] and summarize the following result for the sake of completeness of this paper.

We begin our analysis using the free space channel model shown in Fig. 4. For a single radiation channel, the total

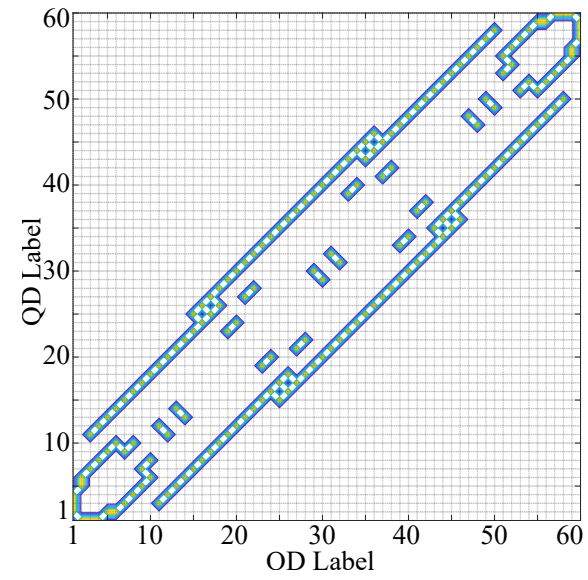

Fig. 5. In this grid arrangement, an interacting pair of QDs is highlighted using a colored box. The presence of these interactions is critical in achieving superradiant emission. The assembly QDs are labeled using the same technique used in Fig 1. Only the neighboring QDs that are highlighted in colored boxes are considered to interact with each other. This implies that the interaction rate matrix $\boldsymbol{\Lambda}_{i n t}$ is sparse and occupies the same rate value at each of the highlighted places. Incorporating interactions beyond this limit will break the symmetry SRC, thus rendering the system subradiant.

emitted power from the $p$ th QD across the spectrum can be expressed by considering the relationships in equations (7) and (8):

$$
\left\langle\mathcal{P}_{(t, \phi)}^{q}\right\rangle=\sum_{p=1}^{N} \sum_{q=1}^{N}\left\langle a_{p}(t)^{*}[\mathcal{E}]_{(p, \phi)}^{T}[\mathcal{E}]_{(q, \phi)} a_{q}(t)\right\rangle .
$$

For convenience, we define: $\mathcal{E}=\Lambda_{E}^{\forall p \rightarrow \forall \phi}$, the matrix already known through equation (8). Now, solving equation (11) depends on deriving the expected value of internal emitter amplitudes at time $t$. Using Fourier relations, we can convert this quantity as follows: $\left\langle a_{p}(t)^{*} a_{q}(t)\right\rangle=$ $\int_{0}^{\infty} \mathrm{d} \omega \int_{0}^{\infty} \mathrm{d} \omega^{\prime}\left(\exp \left[-j\left(\omega-\omega^{\prime}\right) t\right]\left\langle a_{p}(\omega)^{*} a_{q}\left(\omega^{\prime}\right)\right\rangle\right)$. Now we need to solve for $\left\langle a_{p}(\omega)^{*} a_{q}\left(\omega^{\prime}\right)\right\rangle$, which can be done by modifying equation (5). We avoid strenuous recalculations and use the result directly for this derivation. Furthermore, according to Fourier analysis: $\left\langle\mathcal{P}_{(t, \phi)}^{q}\right\rangle=\int_{0}^{\infty} \mathcal{P}_{(\omega, \phi)}^{q} \mathrm{~d} \omega$. Finally, the collective emission from the assembly can be expressed as a summation. Based on these facts, we write:

$$
\mathcal{P}_{(\omega, \phi)}=\frac{\lambda_{0} \Theta(\omega, T)}{4 m \pi^{2} \cos \phi} \sum_{p=1}^{N} \sum_{q=1}^{N} \sum_{r=1}^{N} \mathcal{Q}_{(p, r)}^{*} \mathcal{Q}_{(r, u)} \sqrt{\Lambda_{E}^{p} \Lambda_{E}^{q}},
$$

where the matrix $\mathcal{Q}=\left[[j \boldsymbol{\Omega}+\boldsymbol{\Lambda}]\left[\sqrt{\boldsymbol{\Lambda}_{A}}\right]\right]^{-1}$ results from the expression for $\left\langle a_{p}(\omega)^{*} a_{q}\left(\omega^{\prime}\right)\right\rangle$. Finally, the channel dependence of equation (12) can be omitted by normalizing using the channel flux density [22], which yields the following for the final emission spectrum:

$$
\mathcal{P}_{\omega}=\mathcal{P}_{0} \frac{\lambda_{0}}{2 \pi} \sum_{p=1}^{N} \sum_{q=1}^{N} \sum_{r=1}^{N} \mathcal{Q}_{(p, r)}^{*} \mathcal{Q}_{(r, u)} \sqrt{\Lambda_{E}^{p} \Lambda_{E}^{q}},
$$

where $\mathcal{P}_{0}=\varepsilon_{\omega} \hbar \omega^{2} /\left(4 \pi^{2} c \exp \left(\hbar \omega / k_{B} T\right)-1\right)$ is the power spectral density of a gray-body radiator [22].

This is the final analytical result obtained in this manuscript and can now be substituted in equations (2) and (3) to conduct tissue model simulations. 


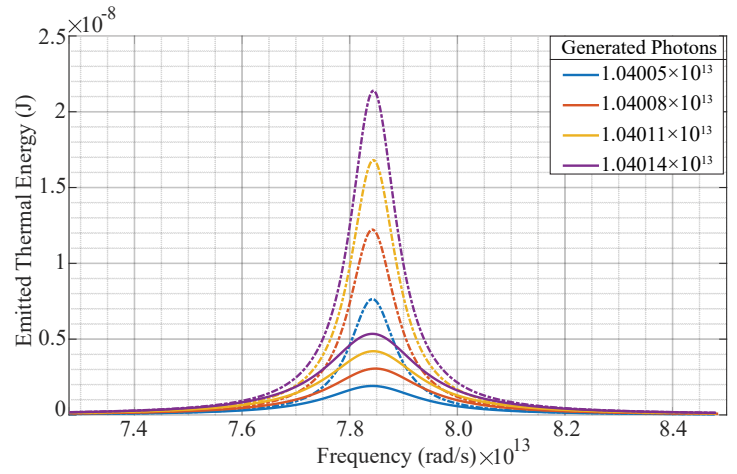

Fig. 6. The emission spectra are plotted using the derived analytical spectrum in equation (13). Continuous and dashed lines correspond to the superradiant and non-interacting assemblies, respectively. The curves correspond to different QD configurations with varying photon generation rates. The noninteracting assembly always outperforms the superradiant counterpart, which is the expected behavior.

\section{RESULTS AND DISCUSSION}

\section{A. Simulation Parameters}

First we analyzed the non-interacting emitter assembly, the dynamics of which are described by equation (2). Here, we assumed that $\Lambda_{\text {int }}(p, b)=0, \forall p, b$ and solved equation (13). A similar approach was used to solve equation (3), where we assumed that $\Lambda_{i n t}(p, b)$ takes the form of the sparse matrix shown in Fig. 5.

We assumed that the initial temperature of both emitter assemblies is at $323.15 \mathrm{~K}$, which is well suited for cancer hyperthermia. This corresponds to a resonance frequency of $7.84 \times 10^{13} \mathrm{rads}^{-1}$ for thermal photons. In the simulation, we assume that all emitters are engulfed within cancerous tissue. Therefore, we assumed the bath permittivity: $\epsilon_{b}=7.17$, which is in agreement with published results [33]. For simplicity, we ignored the temperature and frequency dependencies of $\epsilon_{b}$ for this simulation. In order to perform cancer hyperthermia effectively, we assumed the resultant dipole matrix element of each QD as $3.3336 \times 10^{-28} \mathrm{Cm}$. This value is in agreement with measured values for CdSe QDs, each with a diameter of 5.6nm [25]. The separation between two adjacent QDs was maintained at $4.4 \mathrm{~nm}$, which corresponds to $r=2.2 \mathrm{~nm}$.

For the superradiant assembly, these parameters correspond to a total inter-emitter coupling rate of $1.309 \times 10^{13} \mathrm{~s}^{-1}$ for all interacting QDs. Since we are assuming a 3-nearest neighbor scenario, this value has to be scaled appropriately to find the effective coupling rate $\boldsymbol{\Lambda}_{i n t}$. The value for $\boldsymbol{\Lambda}_{E}=2.618 \times$ $10^{13} \mathrm{~s}^{-1}$ can be directly calculated using equation (10). Due to the lack of experimental data for photon emissivity and absorptivity, we picked a variety of photon generation rates for our simulations. Finally, the value for $\Lambda_{A}$ is found using the principle of energy conservation.

The heat transfer simulation for the tissue model is solved for both transient and steady-states. At $t=0$, the emitters, tumor and healthy tissue regions are assumed to be at $323.15 \mathrm{~K}$, $311.15 \mathrm{~K}$ and $310.5 \mathrm{~K}$, respectively. For both tissue types, thermal conductivity and mass density were maintained at 0.48 $\mathrm{Wm}^{-1} \mathrm{~K}^{-1}$ and $1080 \mathrm{kgm}^{-3}$, respectively. Furthermore, specific heat capacities of 3000 and $3500 \mathrm{Jkg}^{-1} \mathrm{~K}^{-1}$ were maintained for healthy and tumor tissue types, respectively. For the simulation, we placed 8 emitter bundles close to the edge of the tissue region. Each emitter bundle was assumed to comprise 1000 buckyball assemblies. The size of a single emitter assembly was assumed to be negligibly small compared to the size of the tumor and the specific heat capacity of each assembly was assumed to be negligibly small. All emitter parameters were obtained by solving equations (2) and (3) for the non-resonant and superradiant emitter assembly simulations, respectively [21].

However, it is noted that different QD parameters are required for a realistic simulation because the biocompatibility of TOPO-coated CdSe QDs is unestablished. Furthermore, it is necessary to functionalize the surface of each QD prior to in-vivo applications, which will result in further modified QD parameters. Furthermore, we have ignored the temperature dependent variations to these parameters. When simulating the tissue model, we have assumed that the effects of blood perfusion and metabolic heat generation are negligible. We have also assumed that the simulated region is free from blood vessels. Moreover, it is known that the application of hyperthermia will alter the thermal tissue characteristics. We have ignored such changes as well. In fact, the simulated tissue model is a crude approximation, that is presented to juxtapose and validate the superior cancer hyperthermia characteristics of the superradiant assembly in comparison with its conventional counterpart.

\section{B. Emission Characteristics}

As shown in Fig. 6, for the time independent emission, a Lorentzian pattern is observed for both non-resonant and superradiant assemblies. This is the expected result for emissions of this nature [22]. These results are obtained by solving equation (10) for four photon generation rates. For each instance, it is evident that the non-resonant assembly emits a larger number of photons, compared to its superradiant counterpart. This is expected, given that the resonant emitter assembly suffers losses due to inter-emitter interactions.

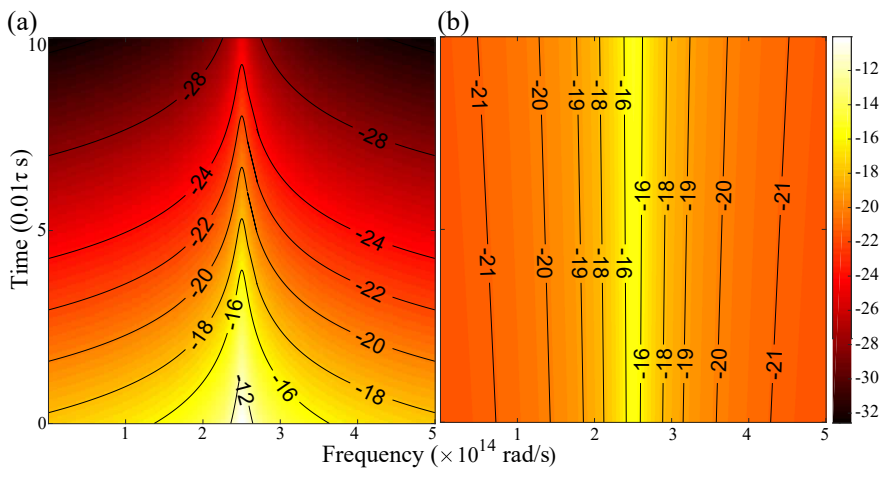

Fig. 7. These plots depict the emission dynamics for two analogous (a) superradiant, (b) non-interacting, emitter assemblies. The photon generation rate is assumed to be $1.04014 \times 10^{13} \mathrm{~s}^{-1}$. These results are obtained by solving (a) equation (3) and (b) equation (2), respectively. Color values correspond to the $\log$ of generated energy at each time and frequency point. The negative values suggest that a single assembly only generates a minute amount of heat. The black contour lines highlight the contrasting emission dynamic patterns of the two emitter assemblies. 


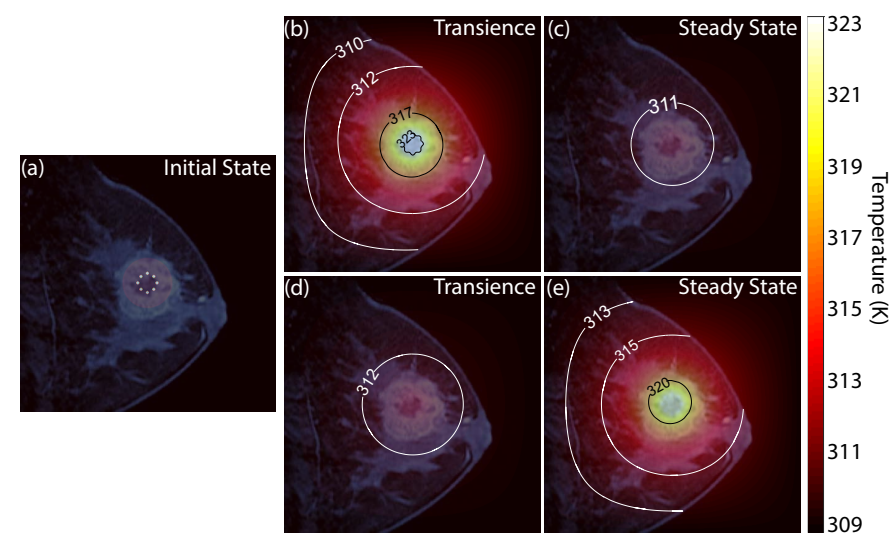

Fig. 8. Artistically illustrated thermal characterization of the non-interacting and superradiant assemblies. (a) The simulation state at $t=0$. Three clearly disparate temperature regions are visualized. The emitters, tumor and healthy tissue are at 323.15, 311.15 and 310.15K, respectively. (b,d) During transience, the superradiant assembly has clearly obtained a sufficiently high temperature within the tumor tissue for cancer hyperthermia. The non-resonant assembly has a lower power emission and the temperature increase is marginal. (c,e) At the steady-state, the superradiant assembly maintains the tumor temperature at around $312 \mathrm{~K}$ while maintaining the healthy tissues at harmless temperatures. In contrast, the non-resonant assembly has increased the temperature of healthy tissues adjacent to the tumor to dangerously high values.

Fig. 7 shows the complete dynamics for the two assemblies. Color values depict the log of generated energy, at each frequency and time point. These results are for the highest photon generation rate shown in Fig. 6. It is noted that, although the superradiant assembly generates a lower number of photons for a given frequency as shown in Fig. 6, the emission power at resonance at the beginning of the superradiant pulse is much higher $\left(\sim \times 10^{4}\right)$ than the power generated by the non-resonant counterpart at any frequency and time point.

\section{Tissue Model Temperature Characteristics}

Initially, it is assumed that there are 3 clearly disparate temperature zones as shown in Fig. 8(a). The tumor region is confined to a circle of radius $15 \mu \mathrm{m}$ and initially maintains a temperature of $311.15 \mathrm{~K}$. The sub-figures throughout Fig. 8 maintain the same scale and the human breast outline is overlaid as a purely artistic illustration and its dimensions are ignored.

At $t=0$, both emitter assemblies have the same conditions as shown in Fig. 8(a). During transience, it is observed that the superradiant assembly is capable of effectively increasing the temperature of the tumor region to around $317 \mathrm{~K}$ as shown in Fig. 8(b). This increase is sufficient for successful cancer hyperthermia. Comparatively, the non-resonant assembly barely increases the tumor temperature beyond its starting value during transience as shown in Fig. 8(d). This result is clearly in agreement with Fig. 7. The high powered and ephemeral superradiant pulse clearly increase the temperature of tumor tissue at the commencement of emission, compared to the conventional counterpart. At steady-state, it is observed that the superradiant assembly barely increases the normal tissue temperature beyond its starting value as shown in Fig. 8(c). Conversely, at steady-state, the conventional assembly has increased the temperature of the adjacent healthy tissue beyond $320 \mathrm{~K}$ as shown in Fig. 8(e). Since the conventional assembly emits a higher number of photons compared to the superradiant counterpart, this result is expected. It is noted that both these results are recorded for the decay of a single energy pulse.

These observations imply that, when using a conventional emitter assembly for cancer hyperthermia, inadvertent hyperthermia of healthy tissues is unavoidable. This is attributed to the slow release of a large number of thermal photons, which leads to undesired temperature rises in adjacent healthy tissues. However, our results suggest that by utilizing a superradiant pulse instead, it is possible to significantly reduce the hyperthermia of adjacent healthy tissues. Therefore, using our proposed assembly to repeatedly deliver a superradiant pulse of heat from within the tumor environment will enhance cancer hyperthermia over using its conventional counterpart.

\section{CONCLUSION}

We have analyzed and illustrated that an assembly of QD thermal emitters, placed at the vertices of a truncated icosahedron, can achieve controlled superradiant emission under the influence of an external electric field. We simulated and proved that under the right conditions, such an emitter assembly can be used to enhance cancer hyperthermia by minimizing the harm to adjacent healthy tissues.

For our analysis, we derived analytical expressions to characterize the dynamics of the emitter assembly. To validate our claims, we used experimental parameters to model a breast cancer environment and conducted thermal simulations. Our results clearly indicate that the superradiant emitter assembly outperforms the conventional, non-resonant counterpart in delivering enhanced cancer hyperthermia by reducing harm to adjacent healthy tissues.

\section{REFERENCES}

[1] J. Van der Zee, "Heating the patient: a promising approach?" Ann. Oncol., vol. 13, no. 8, pp. 1173-1184, 2002.

[2] G. F. Baronzio and E. D. Hager, Hyperthermia in cancer treatment: a primer. Springer Science \& Business Media, 2008.

[3] M. Gross and S. Haroche, "Superradiance: An essay on the theory of collective spontaneous emission," Phys. Rep., vol. 93, no. 5, pp. 301396, 1982.

[4] H. Hapuarachchi, S. Mallawaarachchi, H. T. Hattori, W. Zhu, and M. Premaratne, "Optoelectronic figure of merit of a metal nanoparticlequantum dot (mnp-qd) hybrid molecule for assessing its suitability for sensing applications," J. Phys. Condens. Matter, vol. 30, no. 5, p. 054006, 2018.

[5] T. Warnakula, M. I. Stockman, and M. Premaratne, "An improved scheme for modelling a spaser made of identical gain elements," J. Opt. Soc. Am. B, 2018.

[6] L. Kumarapperuma, M. Premaratne, P. K. Jha, M. I. Stockman, and G. P. Agrawal, "Complete characterization of the spasing (1-1) curve of a three-level quantum coherence enhanced spaser for design optimization," Applied Physics Letters, vol. 112, no. 20, p. 201108, 2018.

[7] Y. Liu, X. Dai, S. Mallawaarachchi, H. Hapuarachchi, Q. Shi, D. Dong, S. H. Thang, M. Premaratne, and W. Cheng, "Poly(nisopropylacrylamide) capped plasmonic nanoparticles as resonance intensity-based temperature sensors with linear correlation," J. Mater. Chem. C, vol. 5, pp. 10926-10932, 2017.

[8] R. H. Dicke, "Coherence in spontaneous radiation processes," Phys. Rev., vol. 93, no. 1, p. 99, 1954.

[9] K. Higgins, S. Benjamin, T. Stace, G. Milburn, B. W. Lovett, and E. Gauger, "Superabsorption of light via quantum engineering," Nat. commun., vol. 5, 2014. 
[10] M. G. Benedict, Super-radiance: Multiatomic coherent emission. CRC Press, 1996.

[11] D. Meiser and M. J. Holland, "Steady-state superradiance with alkalineearth-metal atoms," Phys. Rev. A, vol. 81, no. 3, p. 033847, 2010.

[12] G. L. Celardo, F. Borgonovi, M. Merkli, V. I. Tsifrinovich, and G. P Berman, "Superradiance transition in photosynthetic light-harvesting complexes," J. Phys. Chem. C, vol. 116, no. 42, pp. 22 105-22111, 2012.

[13] J. G. Bohnet, Z. Chen, J. M. Weiner, D. Meiser, M. J. Holland, and J. K. Thompson, "A steady-state superradiant laser with less than one intracavity photon," Nature, vol. 484, no. 7392, pp. 78-81, 2012.

[14] R. A. Molina, E. Benito-Matías, A. D. Somoza, L. Chen, and Y. Zhao, "Superradiance at the localization-delocalization crossover in tubular chlorosomes," Phys. Rev. E, vol. 93, no. 2, p. 022414, 2016.

[15] F. Chung and S. Sternberg, "Mathematics and the buckyball," Am. Sci., vol. 81, no. 1, pp. 56-71, 1993.

[16] M. Scheibner, T. Schmidt, L. Worschech, A. Forchel, G. Bacher, T. Passow, and D. Hommel, "Superradiance of quantum dots," Nat. Phys., vol. 3, no. 2, p. 106, 2007.

[17] Y. Xu, A. Karmakar, D. Wang, M. W. Mahmood, F. Watanabe, Y. Zhang, A. Fejleh, P. Fejleh, Z. Li, G. Kannarpady et al., "Multifunctional fe3o4 cored magnetic-quantum dot fluorescent nanocomposites for $\mathrm{rf}$ nanohyperthermia of cancer cells," J. Phys. Chem. C, vol. 114, no. 11 pp. 5020-5026, 2010.

[18] H. S. Mansur, A. A. Mansur, A. Soriano-Araújo, and Z. I. Lobato, "Beyond biocompatibility: an approach for the synthesis of zns quantum dotchitosan nano-immunoconjugates for cancer diagnosis," Green Chem., vol. 17, no. 3, pp. 1820-1830, 2015.

[19] O. Chen, H. Zhu, Z. Fan, Y. Yuan, M. A. Wilson, K. Hills-Kimball, Z. Wei, J. He, R. Li, and M. Gruenwald, "Self-assembly of quantum dot-gold hetero-dimer nanocrystals with orientational order," Nano Lett., 2018.

[20] M. Creasey, J.-H. Lee, Z. Wang, G. J. Salamo, and X. Li, "Selfassembled ingaas quantum dot clusters with controlled spatial and spectral properties," Nano Lett., vol. 12, no. 10, pp. 5169-5174, 2012.

[21] L. Bezerra, M. Oliveira, T. Rolim, A. Conci, F. Santos, P. Lyra, and R. Lima, "Estimation of breast tumor thermal properties using infrared images," Signal Process., vol. 93, no. 10, pp. 2851-2863, 2013.

[22] M. Zhou, S. Yi, T. S. Luk, Q. Gan, S. Fan, and Z. Yu, "Analog of superradiant emission in thermal emitters," Phys. Rev. B, vol. 92, no. 2, p. 024302, 2015.

[23] B. Coffey and R. Friedberg, "Effect of short-range coulomb interaction on cooperative spontaneous emission," Phys. Rev. A, vol. 17, no. 3, p. 1033, 1978.

[24] Y. Sonnefraud, N. Verellen, H. Sobhani, G. A. Vandenbosch, V. V. Moshchalkov, P. Van Dorpe, P. Nordlander, and S. A. Maier, "Experimental realization of subradiant, superradiant, and fano resonances in ring/disk plasmonic nanocavities," ACS nano, vol. 4, no. 3, pp. 1664 1670,2010

[25] M. Shim and P. Guyot-Sionnest, "Permanent dipole moment and charges in colloidal semiconductor quantum dots," The Journal of chemical physics, vol. 111, no. 15, pp. 6955-6964, 1999.

[26] P. Fry, I. Itskevich, D. Mowbray, M. Skolnick, J. Finley, J. Barker, E. O'Reilly, L. Wilson, I. Larkin, P. Maksym et al., "Inverted electronhole alignment in inas-gaas self-assembled quantum dots," Phys. Rev. Lett., vol. 84, no. 4, p. 733, 2000.

[27] I. A. Ostapenko, G. Hönig, C. Kindel, S. Rodt, A. Strittmatter, A. Hoffmann, and D. Bimberg, "Large internal dipole moment in ingan/gan quantum dots," Appl. Phys. Lett., vol. 97, no. 6, p. 063103, 2010.

[28] N. Bar-Gill, E. E. Rowen, and N. Davidson, "Spectroscopy of strongpulse superradiance in a bose-einstein condensate," Phys. Rev. A, vol. 76, no. 4, p. 043603, 2007.

[29] S. Fan, W. Suh, and J. Joannopoulos, "Temporal coupled-mode theory for the fano resonance in optical resonators," J. Opt. Soc. Am. A, vol. 20, no. 3, pp. 569-572, 2003.

[30] H. A. Haus, Electromagnetic noise and quantum optical measurements. Springer-Verlag, Berlin, 2000.

[31] S. Mallawaarachchi, M. Premaratne, S. D. Gunapala, and P. K. Maini, "Tuneable superradiant thermal emitter assembly," Phys. Rev. B, vol. 95 , no. 15 , p. $155443,2017$.

[32] S. Mallawaarachchi, S. D. Gunapala, M. I. Stockman, and M. Premaratne, "Generalized superradiant assembly for nanophotonic thermal emitters," Phys. Rev. B, vol. 97, no. 12, p. 125406, 2018.

[33] T. Sugitani, S.-i. Kubota, S.-i. Kuroki, K. Sogo, K. Arihiro, M. Okada, T. Kadoya, M. Hide, M. Oda, and T. Kikkawa, "Complex permittivities of breast tumor tissues obtained from cancer surgeries," Appl. Phys. Lett., vol. 104, no. 25, p. 253702, 2014.

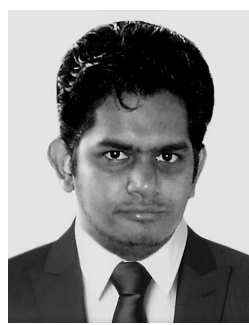

Sudaraka Mallawarachchi received a B.Sc. (Electronic and Telecommunication Engineering) from University of Moratuwa, Sri Lanka ('14). Until '16, he was a Lecturer at the same institute. Since then, he has been working with Prof. Malin Premaratne for his Ph.D. degree in Electrical and Computer Systems Engineering at Monash University. His current research interests include nanobiophotonics, spasers and photothermal cancer therapy with an emphasis on computer modeling and simulations.

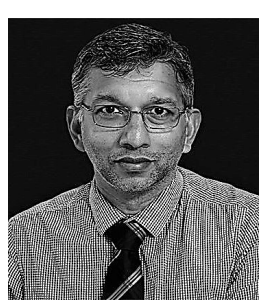

Malin Premaratne received a B.Sc. (Mathematics) \& B.E. (Electrical and Electronics Engineering) with first-class honors and a Ph.D. degree from the University of Melbourne (UoM), in ' 95 and '98, respectively. From '98-'00, he was with the Photonics Research Laboratory at the UoM where he was the co-project leader of the CRC Optical Amplifier project and was associated with Telstra, Australia, and Hewlett Packard, USA. From '01'03, he worked as a consultant to several companies including Cisco, Lucent, Ericsson, Siemens, VPISystems, Telcordia, Ciena, and Tellium. Since '04, he has guided the research program in high-performance computing applications and complex systems simulations at the $\mathrm{A} \chi \mathrm{L}$, Monash University where he is currently the Research Director and a Full Professor. He is also a Visiting Researcher with the Jet Propulsion Laboratory at Caltech, the UoM, Australian National University, UCLA, the University of Rochester New York, and Oxford University. He has authored more than 200 journal papers and a book. He has given presentations on modeling and simulation of optical devices at major international meetings and scientific institutions in the USA, Europe, Asia, and Australia. Currently he is an associate editor for IEEE Photonics Technology Letters, IEEE Photonics Journal, and The Optical Society Advances in Optics and Photonics Journal. He is a Fellow of the Optical Society of America, and The Institute of Engineers Australia.

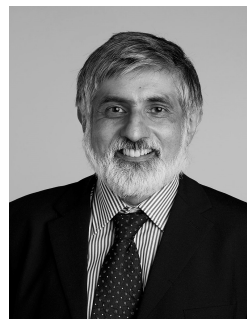

Philip K. Maini received his B.A. (Mathematics) from Balliol College, Oxford, in '82 and his DPhil in '85. In ' 88 he was appointed Asst. Professor in the Mathematics Department at the University of Utah, Salt Lake City. In '90 he returned to Oxford as a University Lecturer and in '98 was appointed Professor of Mathematical Biology by Recognition of Distinction and Director of the WCMB. In '05 he was appointed Statutory Professor of Mathematical Biology. $\mathrm{He}$ is on the editorial boards of a large number of journals, including serving as the Editorin-Chief of the Bulletin of Mathematical Biology ['02-'15]. He is a Fellow of the IMA, a SIAM Fellow, an Inaugural SMB Fellow, a Fellow of the Royal Society of Biology, and Miembro Correspondiente (Foreign Fellow), La Academia Mexicana de Ciencias. In ' 15 he was elected Fellow of the Royal Society, and in '17 he was elected Fellow of the Academy of Medical Sciences, and Foreign Fellow of the Indian National Science Academy. His present research projects include the modelling of avascular and vascular tumours, normal and abnormal wound healing, and a number of applications of mathematical modelling in pattern formation in early development, as well as the theoretical analysis of the mathematical models that arise in all these applications. He has over 300 publications and has held visiting positions at a number of universities worldwide. He was a Distinguished Foreign Visiting Fellow, Hokkaido University ('02). He co-authored with Jonathan Sherratt and Paul Dale a Bellman Prize winning paper ('97), was awarded a Royal Society Leverhulme Trust Senior Research Fellowship for '01-'02 and a Royal Society-Wolfson Research Merit Award ('06-'11). In '09 he was awarded the LMS Naylor Prize and Lectureship and in '14 he was listed in "The World's Most Influential Scientific Minds" (Thomson Reuters). In '07 he was awarded the Arthur T. Winfree Prize from the Society of Mathematical Biology. 